\title{
Nonequilibrium growth of patchy-colloid networks on substrates
}

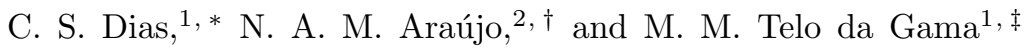 \\ ${ }^{1}$ Departamento de Física, Faculdade de Ciências da Universidade de Lisboa, \\ P-1749-016 Lisboa, Portugal and Centro de Física Teórica e Computacional, \\ Universidade de Lisboa, Avenida Professor Gama Pinto 2, P-1649-003 Lisboa, Portugal \\ ${ }^{2}$ Computational Physics for Engineering Materials, IfB, ETH Zurich, \\ Wolfgang-Pauli-Strasse 27, CH-8093 Zurich, Switzerland
}

\begin{abstract}
Patchy colloids with highly directional interactions are ideal building blocks to control the local arrangements resulting from their spontaneous self-organization. Here we propose their use, combined with substrates and nonequilibrium conditions, to obtain novel structures, different from those of equilibrium thermodynamic phases. Specifically, we investigate numerically the irreversible adhesion of three-patch colloids near attractive substrates, and analyze the fractal network of connected particles that is formed. The network density profile exhibits three distinct regimes, with different structural and scaling properties, which we characterize in detail. The adsorption of a mixture of three- and two-patch colloids is also considered. An optimal fraction of two-patch colloids is found where the total density of the film is maximized, in contrast to the equilibrium gel structures where a monotonic decrease of the density has been reported.
\end{abstract}

PACS numbers: 82.70.Db,07.05.Tp,05.70.Ln

\section{INTRODUCTION}

The past few years have witnessed a sustained interest in the self-organization of patchy colloids, with the development of a wide range of techniques to synthesize them [1] 6]. These particles, with functionalized surfaces, yield new features such as anisotropic interactions, control of the valence, and the formation of permanent electrical dipoles, paving the way to the development of novel materials with fine tuned mechanical, optical, and thermal properties [3, 7, 9]. Understanding how nonequilibrium conditions influence this self-organization is crucial to develop strategies to design new materials, as the novel structures emerge at very low temperatures, where thermal and mechanical equilibration might be difficult to achieve under normal experimental conditions.

Theoretical and experimental studies of patchy colloids have been focused on their cooperative behavior in solution [9 12, where several models were considered with the aim of describing a range of more complex building blocks such as amphiphilic molecules, colloidal clays, proteins, and DNA nano-assemblies [7, 9, 11, 13 18. By contrast, the investigation of self-organization of the simplest of these models at planar substrates is only just beginning [19, 20]. Theoretical studies have shown that, in the presence of a substrate, a rich equilibrium phase diagram emerges with very unusual properties such as, e.g., two wetting transitions and a nonmonotonic surface tension [20. These works have been restricted to equilibrium features; however, it is widely recognized that the use of substrates might improve the degree of control of aggregation, especially under nonequilibrium conditions

\footnotetext{
* csdias@cii.fc.ul.pt

$\dagger$ nuno@ethz.ch

$\ddagger$ margarid@cii.fc.ul.pt
}

Interaction range

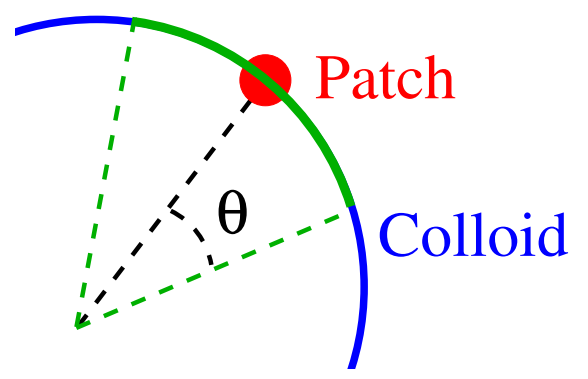

FIG. 1. (color online) Schematic representation of a patch (red) on the surface of a colloid (blue) and its interaction range (green). The limits of the interaction range are defined by an angle $\theta$ with the center of the patch.

21, 22]. For example, a growth direction can be defined which allows the use of kinetic features to control the film structure 23 .

In this paper we address the nonequilibrium adsorption of patchy colloids on substrates, resulting from the irreversible nature of the binding, and we characterize the resulting network of connected particles. In deep contrast with equilibrium films, a fractal network is assembled with a fractal dimension compatible with the one reported for Diffusion Limited Aggregation (DLA) [24, 25]. We systematically analyze the dependence of the network structure on the substrate size and diffusion coefficient of the colloids in solution (which can be controlled experimentally, for example, by the thermostat temperature). We show that, although the density of the film strongly depends on the diffusion coefficient, the fractal dimension is resilient over a wide range of growth conditions. By contrast with previous models based on DLA, here directional interactions are considered with 


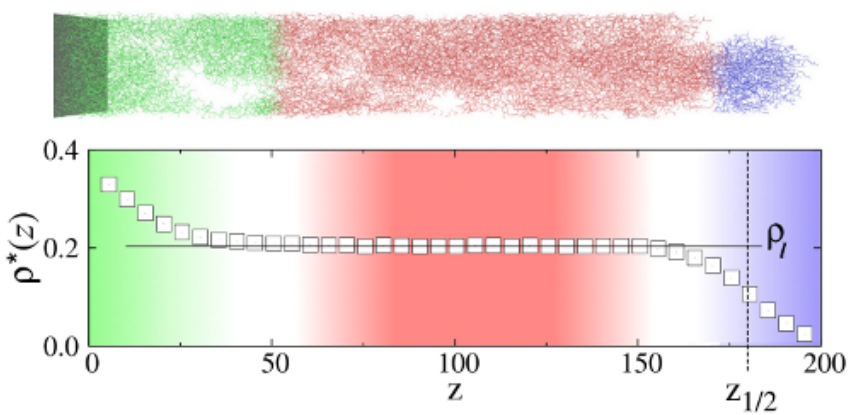

FIG. 2. (color online) Density profile of the colloidal network on a substrate showing three different regimes: surface layer (green, left); liquid film (red, middle); interfacial region (blue, right). Top: Snapshot of a typical configuration, where each stick corresponds to a colloid-colloid connection. Bottom: Density, $\rho^{*}(z)$, as a function of the height, $z$, after the adsorption of 40 particle layers, averaged over 500 independent realizations. $\rho_{l}$ is the density of the liquid film and $z_{1 / 2}$ is the film thickness, defined as the height at which the density is $\rho_{l} / 2$.

results that depend on the diffusion coefficient and colloidal valence.

It has been shown that the distribution of patches affects the aggregation process and, consequently, the equilibrium bulk structures $[8,10,12,18,26,27$. For example, control of the valence allows tuning up the density and temperature of both the gas-liquid and sol-gel critical points [10, 28, 29]. Here, we consider three-patch colloids and investigate the density profile of the adsorbed nonequilibrium network. We then proceed to investigate the adsorption of three- and two-patch colloids, which can be synthesized with the available experimental techniques [2, 7, 30 33, and characterize the dependence of the film density on the concentration of the mixture. Studies of the coexisting thermodynamic structures of mixtures of two- and three-patch colloids in solution reveal a monotonic decrease of the density with the fraction of two-patch colloids [27, 28, 34. Somewhat surprisingly, we have found that the density of the adsorbed network film, under nonequilibrium conditions, increases with the concentration of two-patch particles before it decreases, exhibiting a well defined maximum at an intermediate concentration.

In the following section we describe the model. In Sec. III, we quantify these results and illustrate the full scaling behavior of the nonequilibrium adsorbed film. Finally, in Sec. [V] we draw some conclusions.

\section{MODEL}

In order to simulate the colloidal adsorption we propose and use a stochastic model based on the nonequilibrium Monte Carlo (MC) method. Patchy colloids are frequently described as spherical particles with a short-

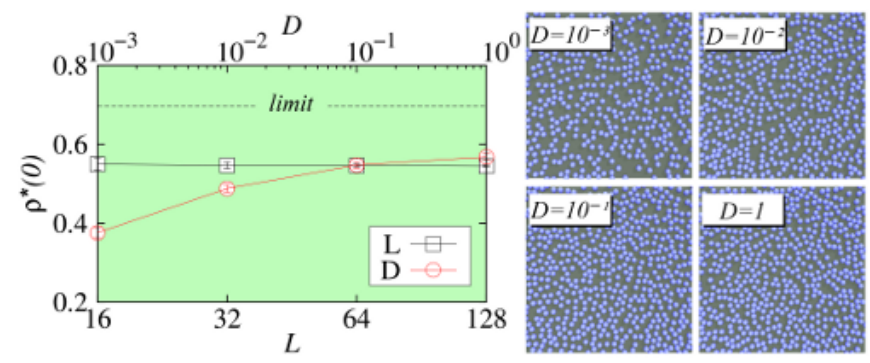

FIG. 3. (color online) Right: Dependence of the density of the first adsorbed layer, $\rho^{*}(0)$, on $D$, for values ranging from $10^{-3}$ to 1 , and substrates with lateral size, $L$, ranging from 16 to 128. Right: Snapshots of the first adsorbed layer (directly in contact with the substrate) for different diffusion coefficients, $D$, namely, $10^{-3}, 10^{-2}, 10^{-1}$, and 1 , on a substrate with lateral size 32 in units of the particle diameter.

range repulsive core and patch-patch attractive interactions. The attraction is truncated at a certain angle around the center of the patch [35].

As show in Fig. 1, to account for particle-particle interaction we define an interaction range (green), on the surface of the colloid (blue), around each patch (red). This range is characterized by a single parameter, namely, the angle $\theta$ with the center of the patch. Here we have used $\theta=\pi / 6$. In the event of a collision with a preadsorbed colloid, if the contact point is within the interaction range of the pre-adsorbed particle, the binding is successful with probability $p$, corresponding to the fraction of the surface of the landing colloid covered by the interaction range of all patches. In the case of successful binding, the position of the landing particle is adjusted based on the patch-patch orientation, otherwise, an elastic collision occurs. The interaction range accounts for both the extension of the patch on the colloid surface and the range of the patch-patch interaction. In particular, the range of the patch-patch interaction is $\pi \sigma / 6$, where $\sigma$ is the colloid diameter. Thus, our model contains a single control parameter (the interaction range) that can be tuned to mimic different patch-patch potentials.

In the presence of a substrate, two characteristic timescales can be identified: one related to the flux of colloids towards the substrate (inter-arrival time) and the other to the binding between patches (binding time). In general, the inter-arrival time is a function of the colloid shape and radius, diffusion coefficient, and concentration of colloids in the bulk. For simplicity, we consider equisized spherical particles and the limit of highly diluted colloids. In this limit, the diffusion coefficient affects only the trajectory of the colloids and the inter-arrival time can be considered much larger than the binding time. Since the binding is irreversible, we assume that the colloids arrive one at a time towards the substrate and adhere instantaneously. In addition, we consider chemical bonds between the patches, which are highly directional and assumed irreversible within the timescale of inter- 


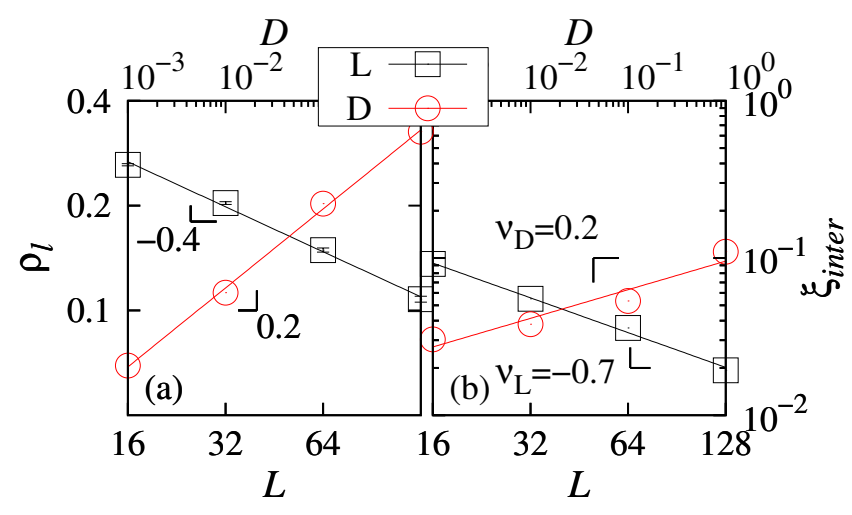

FIG. 4. (color online) (a) Liquid-film density for substrates with lateral size ranging from 16 to 128 , and diffusion coefficient, $D$, between $10^{-3}$ and 1 . (b) Dependence of the interfacial decay length $\xi_{\text {inter }}$ on $L$ and $D$.

est. In order to describe the colloid motion in solution, a Brownian algorithm is considered, adapting ideas previously implemented for Molecular Dynamics (see, e.g. Ref. [10]). Collisions with the solvent are assumed Poisson processes, i.e., the time between collisions is exponentially distributed. At each particle-solvent collision, a new value for the particle velocity is generated, drawn randomly from the Maxwell-Boltzmann distribution at the thermostat temperature. The direction of the velocity is obtained from a uniform distribution. By selecting the thermostat temperature and the collision rate (Poisson process) we can then adjust the diffusion coefficient. The substrate is considered attractive and, therefore, collisions with the substrate always result in irreversible colloidal binding.

Our model shares important features with Diffusion Limited Deposition (DLD), an extension of the famous DLA developed to account for the growth of films on fibers and surfaces 24. In DLD, particles diffuse one after the other and adhere to the first pre-adsorbed particle. For patchy colloids, since a link is established only when there is an effective overlap between interaction ranges, the colloid does not, necessarily, bind during the first collision. Besides, somewhat artificially, in the continuum version of DLD the random walkers' mean free path is considered of uniform length (typically of the order of the diameter of the particle) and, consequently, the diffusion coefficient only affects the time scale. Here, we use a more realistic description where the inter-collision time with the solvent, and consequently the mean free path, is a function of the thermostat temperature. As we will show below, an interesting dependence on the diffusion coefficient emerges.

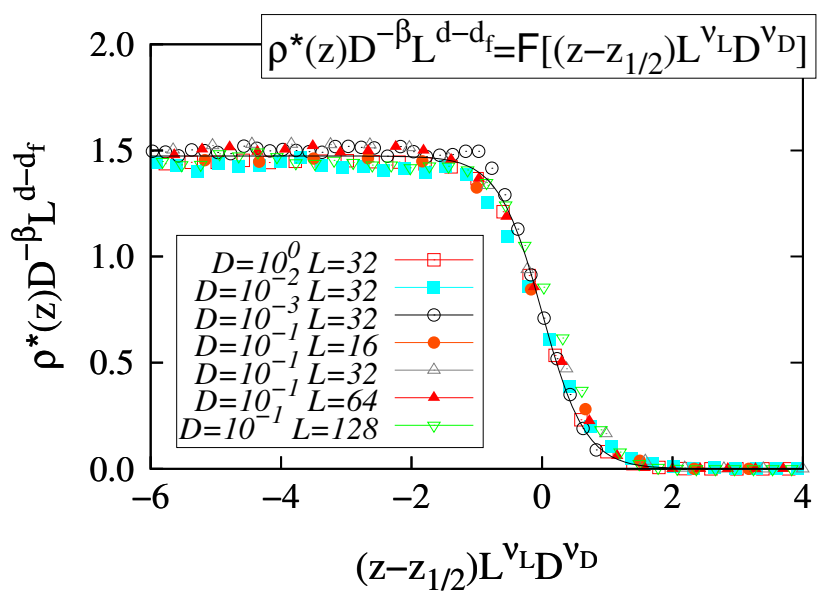

FIG. 5. (color online) Data collapse for the liquid film and interfacial region, including the dependence on the substrate lateral size $L$ and the diffusion coefficient $D$. Results obtained after adsorbing 60 particle layers and averaging over 500 samples for $L=\{16,32\}, 200$ samples for $L=64$, and 100 samples for $L=128$.

\section{RESULTS}

We start with an empty planar square substrate, with lateral size $L$, defined in units of the particle diameter, and assume periodic boundary conditions in the horizontal plane. Iteratively, spherical colloids, with three patches uniformly distributed on their surface, are released one after the other and diffuse until they bind either to the substrate or to a previously adsorbed colloid. Figure 2 shows a snapshot of a typical network of connected three-patch colloids and the density profile $\rho^{*}(z)$, defined as the number of particles per unit volume, where $z$ is the distance to the substrate. One can distinguish three different regimes: surface layer, liquid film, and interfacial region. As we move away from the surface, the density rapidly decreases (surface layer, $z<50$ ) until a saturation value $\rho_{l}$, which is constant within the liquid film $(50<z<150)$, and vanishes in the interfacial region $(z>150)$. In the following, we discuss in detail each of these regimes.

Surface layer. As the colloid-substrate interaction is isotropic, the patches of colloids directly adsorbed on the substrate are oriented randomly. By contrast, the colloid-colloid (i.e., patch-patch) interaction is strongly anisotropic and the network chains extend only along the direction of the patches. As the front of the network propagates, its branches screen the inner layers and span along the lateral direction, with a consequent decrease of the density. This effect was also reported for DLD, where a power-law decay with $z$ is observed, with multifractal scaling [24, 36]. However, here the conspiracy between the order promoted by the patches and the disorder imposed by the first layer results in an exponential decrease, at least for the lateral sizes that were investigated. 


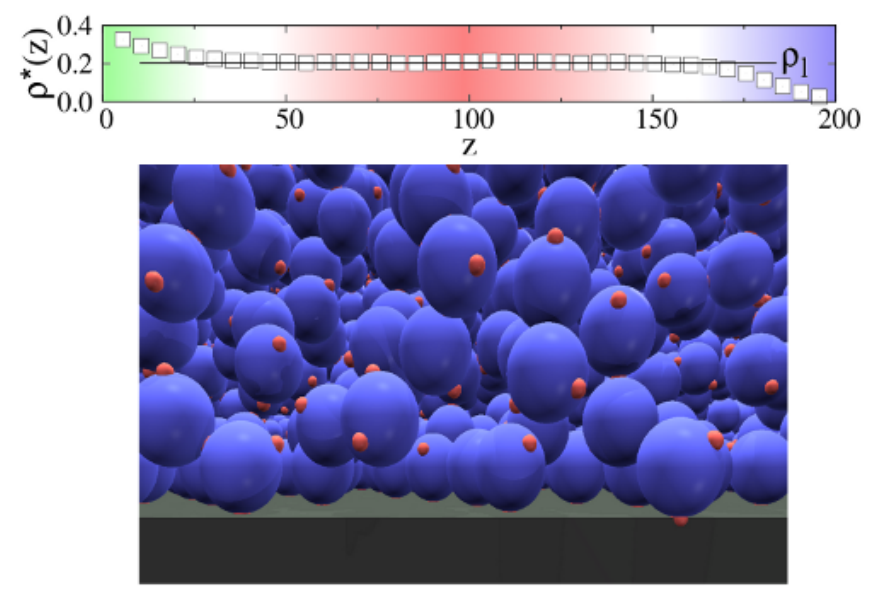

FIG. 6. (color online) Top: Density profile of the colloidal network on a substrate with anisotropic particle-substrate interaction. Results after the adsorption of 40 particle layers, averaged over 500 independent realizations. $\rho_{l}$ is the density of the liquid film. Bottom: Snapshot of the particles in the surface layer regime.

The plot in Fig. 3 shows the dependence of the firstlayer density, $\rho^{*}(0)$, on the size of the substrate $L$ and diffusion coefficient $D$. While there is no significant finitesize effect, an increase of $\rho^{*}(0)$ with $D$ is observed (see snapshots in the same figure). For the irreversible adhesion of colloids on substrates, in the limit where particles only stick to the substrate and not on top of other particles, extensive simulations have shown no significant dependence on the diffusion coefficient 37, 38. In that case, the structure of the film should resemble that of Random Sequential Adsorption (RSA) [21, 39, 40. Instead, the patch-patch interaction promotes the formation of colloid chains hindering the access to the substrate. Additionally, in the diffusion process, the typical colloid mean free path increases with $D$. Since multiple collisions can occur before irreversible binding, the larger the mean free path the higher the probability that the colloids can squeeze into the fjords and, eventually, arrive at the substrate. Consequently, the first-layer density increases with $D$ towards the RSA limit (limit line in Fig. 3). However, patchy particle systems will always form networks and the RSA limit is never reached.

Liquid film. As the lateral growth of the network proceeds, the finite size of the substrate induces a saturation of the density at $\rho=\rho_{l}$. For the range of values of $L$ and $D$ considered here, the network is always a fractal with fractal dimension $d_{f}=2.58 \pm 0.04$, calculated using the box counting algorithm; this value is compatible with the one reported for DLA and DLD 24. Notwithstanding, $\rho_{l}$ depends on $L$ and $D$ (see Fig. 4(a)). In both cases, a power-law scaling is observed, namely, $\rho_{l} \sim L^{d_{f}-d} D^{\beta}$, with $d_{f}-d=-0.4 \pm 0.1$ and $\beta=0.23 \pm 0.02$. While the exponent in the size dependence results from the scale invariance of the network and is straightforwardly con-

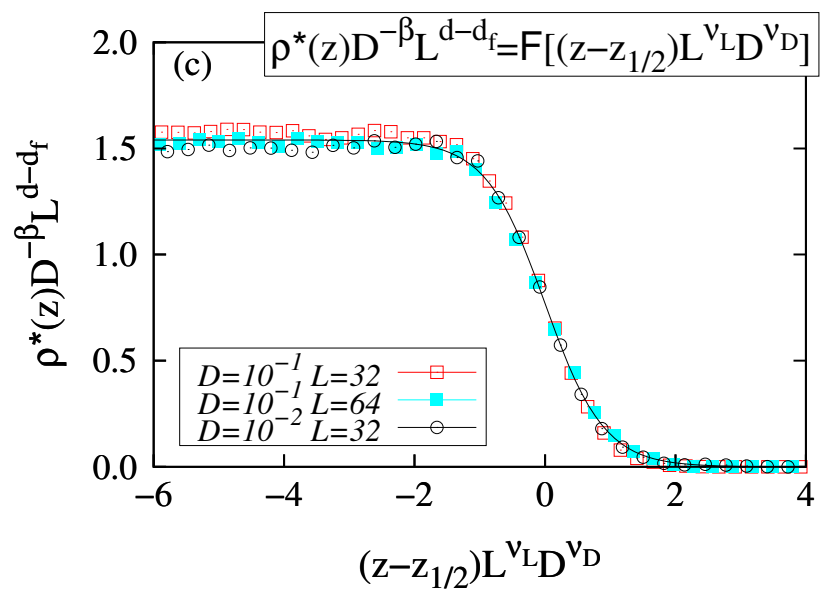

FIG. 7. (color online) Data collapse for the liquid film and interfacial region, where bonds are considered non-optimal. Results are obtained after adsorbing 60 particle layers and averaging over 500 samples for $L=32$ and 200 for $L=64$.
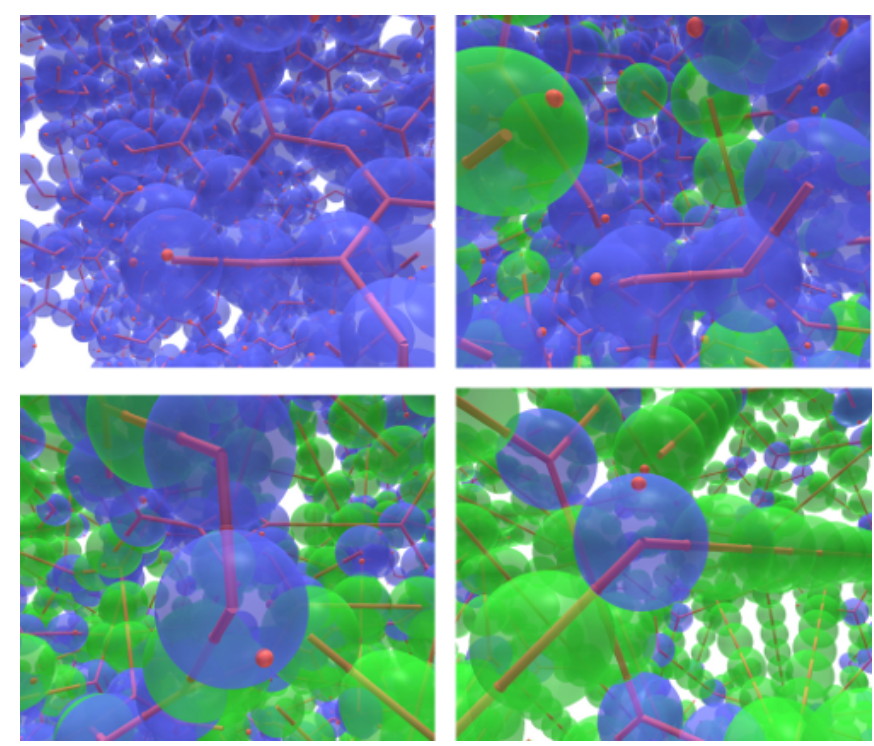

FIG. 8. (color online) Snapshots of a region in the liquid film for different fractions of two-patch colloids, $r_{D}$. From left to right, top to bottom: $0,0.3,0.6$, and 0.8. Snapshots obtained from the adsorption on a substrate with a lateral size of 32 particle diameters and 40 particle layers. Threepatch colloids are in blue (dark), two-patch colloids are in green (light), the (red) spheres on the surface of the colloids represent the patches, and the (red) sticks are the connections between colloids.

nected with $d_{f}$, the dynamic exponent $\beta$ is new and there is no equivalent in previously studied models.

Interfacial region. The density vanishes at the interface between the network and the solution, which corresponds to the active front of the film. The position of the interface depends on the total number of adsorbed particle layers $N$. We define $z_{1 / 2}$ as the 


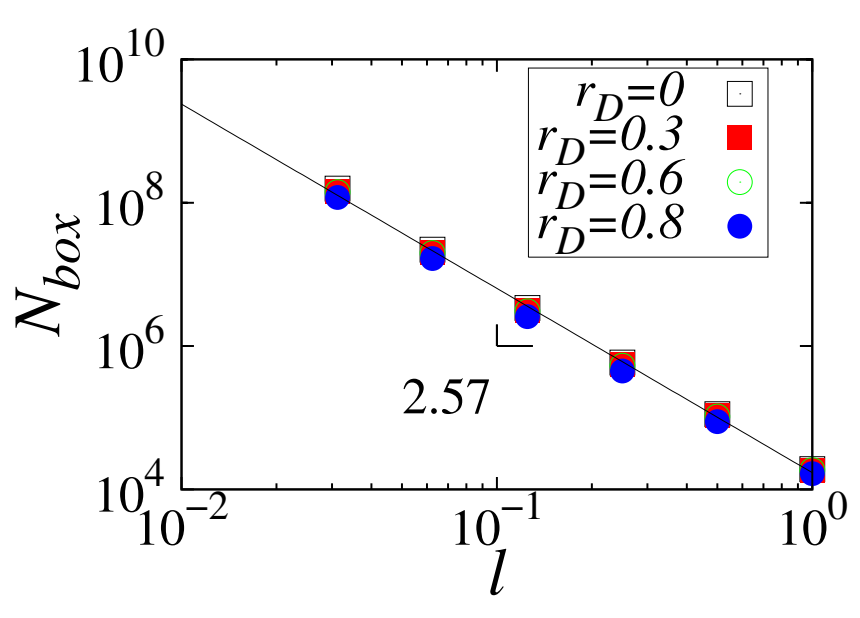

FIG. 9. (color online) Mass scaling as a function of the inverse box size in the box counting algorithm, for networks, with different concentrations of two-patch colloids, $r_{D}$. Results obtained with 32 layers of the 60 layers of adsorbed particles in the liquid film on a substrate with $L=32$, averaged over 20 samples. A fractal dimension $d_{f}=2.57 \pm 0.04$ was found.

thickness of the film, corresponding to the height at which the density is $\rho_{l} / 2$ (see Fig. 2). Since the thickness of the surface layer does not depend on $N, z_{1 / 2}$ asymptotically scales as $z_{1 / 2} \sim N / \rho_{l}=N L^{d-d_{f}}$. The profile of the density in the interfacial region scales as $\rho^{*}(z)=\rho_{l} \tanh \left[\left(z-z_{1 / 2}\right) \xi_{\text {inter }}\right]$, as typically observed for models of diffusion limited growth in the stationary regime (see, e.g., Ref. [41]). The decay exponent $\xi_{\text {inter }}$ also scales with $L$ and $D$ (see Fig. 4(b)). In both cases, a power law is observed, $\xi_{\text {inter }} \sim L^{\nu_{L}} D^{\nu_{D}}$, with $\nu_{L}=-0.73 \pm 0.04$ and $\nu_{D}=0.18 \pm 0.04$.

Based on the properties of the liquid film and interfacial region, we propose full scaling of the density profile with $L$ and $D$, defined as $\rho^{*}(z)=D^{\beta} L^{d_{f}-d} F\left[\left(z-z_{1 / 2}\right) L^{\nu_{L}} D^{\nu_{D}}\right]$, where $F[x]$ is a scaling function described by the hyperbolic tangent. Figure 5 shows the data collapse for several values of $D$ and $L$. This scaling allows the definition of the network density for any size of the substrate and of the diffusion coefficient.

For simplicity, we have considered an isotropic particlesubstrate interaction. In general, some anisotropy is expected due to the patches. Figure 6 shows the density profile of the colloidal network when this interaction is anisotropic. In particular, as illustrated in the snapshot (bottom of the figure), we considered the case where adsorbing colloids bind to the substrate only through the patches. The parameters are those considered in Fig. 2. We show that the qualitative picture discussed here is not affected by the details of the interaction with the substrate and the quantitative results are different only within the surface layer.

We have also assumed optimal bonds such that bind-
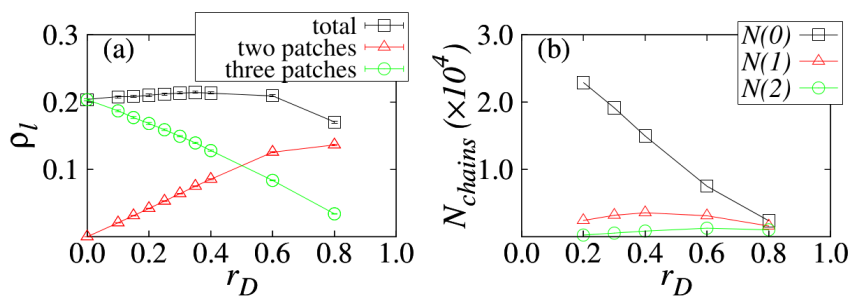

FIG. 10. (color online) Analysis of the dependence on the fraction of two-patch colloids, $r_{D}$. (a) Liquid-film density (black squares) and the contribution from two- (red triangles) and three-patch (green spheres) colloids. (b) Total number of chains of two-patch colloids between three-patch ones with sizes 0,1 , and 2 . The peak of the distribution of chains of unit size coincides with the peak of the film density. Results for 60 particle layers adsorbed on a substrate with $L=32$ averaged over 500 samples.

ing is established along the direction of the patches. It has been shown recently that non-optimal bonds may enrich the diagram of self-organized states [42]. In Fig 7, we also consider the case of non-optimal bonds, i.e., as in the optimal case a landing patchy colloid only sticks to a previously adsorbed one when their interaction ranges overlap, but the binding is established at the contact point. In spite of changes in the liquid film density, the same scaling and fractal dimensions are found.

Adsorption of two- and three-patch colloids. Finally we consider the adsorption of a mixture of two- and threepatch colloids. While the two-patch colloids (patches on the poles) favor long chains, the three-patch particles promote branching. We define $r_{D}$ as the fraction of adsorbed two-patch colloids and investigate how it affects the network structure. Figure 8 shows snapshots of the liquid film for different $r_{D}$. The larger $r_{D}$ the longer the chains of two-patch colloids. In all cases, including $r_{D}=0$, the resulting network is a fractal with the same fractal dimension (see Fig. 9). However, the density of the liquid film can be controlled with $r_{D}$ (see Fig. 10(a)).
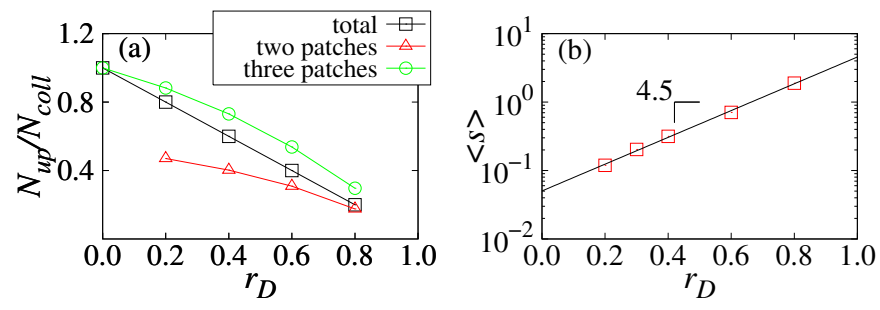

FIG. 11. (color online) (a) Fraction of free (unconnected) patches on two-patch (red triangles) and three-patch (green circles) colloids in the liquid film. (b) Exponential increase of the average size of chains of two-patch colloids.

Somewhat surprisingly, a maximum is observed in the density at a fraction of two-patch particles around 0.35, by contrast to equilibrium coexisting gels (i.e. optimal networks at zero pressure) where a monotonic decrease 
is observed 28. In fact, the film density remains above the three-patch colloid limit $\left(r_{D}=0\right)$ over a wide range of $r_{D}$ (up to around 0.6). As $r_{D}$ increases, long chains of two-patch colloids are formed and the density is expected to decrease. However, in the absence of relaxation, kinetically trapped structures are obtained and geometrical constraints hinder the access of colloids to the freepatches in the inner layers. The competition between the formation of long chains and the maximization of accessible patches drives the reported film density maximum. The plot in Fig. 10(b) shows the number of chains of twopatch colloids of size 0,1 , and 2 . With increasing $r_{D}$, the number of chains of size 0 (corresponding to pairs of connected three-patch colloids) decreases and a maximum in the number of chains of size 1 is observed at the same concentration as the maximum in the film density. In Fig 11 we show that, increasing $r_{D}$ reduces the number of free patches in the film and increases the average size of the chains exponentially.

\section{CONCLUSIONS}

This work reveals that, in the presence of a substrate and under nonequilibrium conditions, new self-organized patterns are obtained which differ from the thermodynamic optimal networks or equilibrium coexisting gels (at zero pressure). For mixtures of three- and two-patch colloids, fractal networks of connected particles are formed with a fractal dimension resilient over a wide range of diffusion coefficients and concentration of two-patch col- loids. Yet, the obtained structures depend both on the diffusion coefficient and the colloidal valence. These networks might be of relevance in the fields of microfluidics and filtering. For example, the density variation in the surface layer resembles the filtering mechanism recently found in the human airways [43. Here, we focused on the nonequilibrium properties of the network, resulting from the irreversible nature of the binding, which dominates at low temperatures. However, in a significant long timescale or at higher temperatures, an adsorbed colloid might detach and rebind to another patch or to the substrate. In this case, the thermodynamic equilibrium structures might, in principle, be reached. As a follow up, the stability and aging of the networks should be investigated as well as the kinetic pathways towards the thermodynamic equilibrium structures. Additionally, techniques to stabilize these structures over extended periods of time might also be a focus of future research. The model may be used to investigate other features such as different particle-substrate interactions [6, 22] and nonoptimal bonds, where fluctuations in the bonding direction are taken into account 42 .

\section{ACKNOWLEDGMENTS}

We acknowledge financial support from the Portuguese Foundation for Science and Technology (FCT) under Contracts nos. EXCL/FIS-NAN/0083/2012, PEstOE/FIS/UI0618/2011, and PTDC/FIS/098254/2008, and also helpful comments from Francisco de los Santos. This work was also supported (NA) by grant number FP7-319968 of the European Research Council
[1] H. C. Shum, A. R. Abate, D. Lee, A. R. Studart, B. Wang, C.-H. Chen, J. Thiele, R. K. Shah, A. Krummel, and D. A. Weitz, Macromol. Rapid Commun. 31, 108 (2010).

[2] E. Duguet, A. Désert, A. Perro, and S. Ravaine, Chem. Soc. Rev. 40, 941 (2011).

[3] S. Sacanna and D. J. Pine, Curr. Opin. Coll. Interf. Sci. 16, 96 (2011).

[4] Z. He and I. Kretzschmar, Langmuir 28, 9915 (2012).

[5] J. Hu, S. Zhou, Y. Sun, X. Fang, and L. Wu, Chem. Soc. Rev. 41, 4356 (2012).

[6] O. I. Wilner and I. Willner, Chem. Rev. 112, 2528 (2012).

[7] A. B. Pawar and I. Kretzschmar, Macromol. Rapid Commun. 31, 150 (2010).

[8] I. Kretzschmar and J. H. K. Song, Curr. Opin. Coll. Interf. Sci. 16, 84 (2011).

[9] E. Bianchi, R. Blaak, and C. N. Likos, Phys. Chem. Chem. Phys. 13, 6397 (2011).

[10] J. Russo, P. Tartaglia, and F. Sciortino, J. Chem. Phys. 131, 014504 (2009).

[11] F. Sciortino and E. Zaccarelli, Curr. Opin. Solid State Mater. Sci. 15, 246 (2011).

[12] F. Sciortino, E. Bianchi, J. F. Douglas, and P. Tartaglia, J. Chem. Phys. 126, 194903 (2007).

[13] Z. Zhang and S. C. Glotzer, Nano Lett. 4, 1407 (2004).
[14] J. P. K. Doye, A. A. Louis, I.-C. Lin, L. R. Allen, E. G. Noya, A. W. Wilber, H. C. Kok, and R. Lyus, Phys. Chem. Chem. Phys. 9, 2197 (2007).

[15] F. Sciortino, A. Giacometti, and G. Pastore, Phys. Chem. Chem. Phys. 12, 11869 (2010).

[16] S. C. Glotzer and J. A. Anderson, Nature Mater. 9, 885 (2010).

[17] G. Rosenthal and S. H. L. Klapp, J. Chem. Phys. 134, 154707 (2011).

[18] B. Ruzicka, E. Zaccarelli, L. Zulian, R. Angelini, M. Sztucki, A. Moussaïd, T. Narayanan, and F. Sciortino, Nature Mater. 10, 56 (2010).

[19] N. Gnan, D. de Las Heras, J. M. Tavares, M. M. Telo da Gama, and F. Sciortino, J. Chem. Phys. 137, 084704 (2012).

[20] N. R. Bernardino and M. M. Telo da Gama, Phys. Rev. Lett. 109, 116103 (2012).

[21] A. Cadilhe, N. A. M. Araújo, and V. Privman, J. Phys.: Condens. Matter 19, 065124 (2007).

[22] N. A. M. Araújo, A. Cadilhe, and V. Privman, Phys. Rev. E 77, 031603 (2008).

[23] T. L. Einstein and T. J. Stasevich, Science 327, 423 (2010).

[24] P. Meakin, Fractals, scaling and growth far from equilibrium (Cambridge Univ Pr, Cambridge, 1998). 
[25] T. A. Witten Jr. and L. M. Sander, Phys. Rev. Lett. 47, 1400 (1981).

[26] E. Bianchi, P. Tartaglia, E. La Nave, and F. Sciortino, J. Phys. Chem. B 111, 11765 (2007).

[27] E. Bianchi, P. Tartaglia, E. Zaccarelli, and F. Sciortino, J. Chem. Phys. 128, 144504 (2008).

[28] E. Bianchi, J. Largo, P. Tartaglia, E. Zaccarelli, and F. Sciortino, Phys. Rev. Lett. 97, 168301 (2006).

[29] J. Russo and F. Sciortino, Phys. Rev. Lett. 104, 195701 (2010).

[30] Y.-S. Cho, G.-R. Yi, J.-M. Lim, S.-H. Kim, V.-N. Manoharan, D. J. Pine, and S.-M. Yang, J. Am. Chem. Soc. 127, 15968 (2005).

[31] A. van Blaaderen, Nature 439, 545 (2006).

[32] D. J. Kraft, R. Ni, F. Smallenburg, M. Hermes, K. Yoon, D. A. Weitz, A. van Blaaderen, J. Groenewold, M. Dijkstra, and W. K. Kegel, Proc. Nat. Acad. Sci. 109, 10787 (2012).

[33] Y. Wang, D. R. Breed, V. N. Manoharan, L. Feng, A. D. Hollingsworth, M. Weck, and D. J. Pine, Nature 491,
$51(2012)$.

[34] H. Liu, S. K. Kumar, F. Sciortino, and G. T. Evans, J. Chem. Phys. 130, 044902 (2009).

[35] G. Villar, A. W. Wilber, A. J. Williamson, P. Thiara, J. P. K. Doye, A. A. Louis, M. N. Jochum, A. C. F. Lewis, and E. D. Levy, Phys. Rev. Lett. 102, 118106 (2009).

[36] A. Coniglio and M. Zannetti, Physica A 163, 325 (1990).

[37] B. Senger, J. C. Voegel, P. Schaaf, A. Johner, A. Schmitt, and J. Talbot, Phys. Rev. A 44, 6926 (1991).

[38] B. Senger, P. Schaaf, J. C. Voegel, A. Johner, A. Schmitt, and J. Talbot, J. Chem. Phys. 97, 3813 (1992).

[39] J. W. Evans, Rev. Mod. Phys. 65, 1281 (1993).

[40] V. Privman, J. Adhesion 74, 421 (2000).

[41] J. B. Collins and H. Levine, Phys. Rev. B 31, 6119 (1985).

[42] F. Romano and F. Sciortino, Nat. Comm. 3, 975 (2012).

[43] B. Button, L.-H. Cai, C. Ehre, M. Kesimer, D. B. Hill, J. K. Sheehan, R. C. Boucher, and M. Rubinstein, Science 337, 937 (2012). 